\title{
Resolution of nonlinear interval problems using symbolic interval arithmetic
}

\author{
Luc Jaulin*, Gilles Chabert \\ ENSIETA, 2, rue François Verny, 29200 Brest, France
}

\section{A R T I C L E I N F O}

\section{Article history:}

Received 17 April 2009

Accepted 24 June 2009

Available online 19 September 2009

Keywords:

Interval analysis

Quantified problems

Symbolic intervals

Constraint propagation

Nonlinear problems

\begin{abstract}
A B S T R A C T
An interval problem is a problem where the unknown variables take interval values. Such a problem can be defined by interval constraints, such as "the interval $[a, b] \subset[a, b]^{2}$ ". Interval problems often appear when we want to analyze the behavior of an interval solver. To solve interval problems, we propose to transform the constraints on intervals into constraints on their bounds. For instance, the previous interval constraint $[a, b] \subset[a, b]^{2}$ can be transformed into the following bound constraints " $a \geq \min \left(a^{2}, a b, b^{2}\right)$ and $b \leq \max \left(a^{2}, a b, b^{2}\right) "$. Classical interval solvers can then be used to solve the resulting bound constraints. The procedure which transforms interval constraints into equivalent bound constraints can be facilitated by using symbolic interval arithmetic. While classical intervals can be defined as a pair of two real numbers, symbolic intervals can be defined as a pair of two symbolic expressions. An arithmetic similar to classical interval arithmetic can be defined for symbolic intervals. The approach will be illustrated on several applications.
\end{abstract}

(c) 2009 Elsevier Ltd. All rights reserved.

\section{Introduction}

Interval analysis (Moore, 1979) is an efficient numerical tool to solve nonlinear problems such as global optimization (Hansen, 1992), set characterization (Jaulin et al., 2001), etc. in a reliable way. Although interval methods made it possible to solve efficiently a large class of nonlinear punctual problems (i.e., problems where the solutions to be found are vectors or real numbers), they also brought new questions and new problems about the properties and the behaviors of the interval algorithms. Most of these new problems can be cast into the framework of interval problems, i.e., problems where the solution set is composed with intervals or boxes.

This paper introduces symbolic intervals with its arithmetic. The idea is similar to that of numerical interval computation: the interval operations are replaced by operations on their bounds. But for symbolic intervals, these operations are performed in a symbolic way. This symbolic arithmetic will make possible to transform an interval problem into a punctual problem in a systematic way. The resulting punctual problem will then be solved using classical numerical interval methods.

To our knowledge, the idea of applying interval arithmetic rules in a symbolic way has never been proposed before. Of course, in the context of interval methods, classic symbolic

\footnotetext{
* Corresponding author. Tel.: +330298348910.

E-mail address: jaulinlu@ensieta.fr (L. Jaulin).

URL: www.ensieta.fr/jaulin/.
}

calculus was already used to improve the efficiency of interval solvers (see, e.g., van Emden, 1999), but the interval rules was only applied on the numerical resolution, not on the symbolic part.

Section 2 shows how an interval problem can be transformed into an equivalent punctual problem. Symbolic interval arithmetic is introduced in Section 3. Section 4 presents some potential applications of symbolic intervals. Most of them cannot be solved with existing tools, to our knowledge. Section 5 concludes the paper.

\section{Interval problem and bound problem}

\subsection{Interval constraint}

An interval $[x]$ is a closed bounded set of $\mathbb{R}$. The set of all intervals is denoted by $\mathbb{R}$. A box $[\mathbf{x}]=\left[x_{1}\right] \times \cdots \times\left[x_{1}\right]$ of $\mathbb{R}^{n}$ is the Cartesian product of $n$ intervals. The set of all boxes of $\mathbb{R}^{n}$ is denoted by $\square \mathbb{R}^{n}$. An interval constraint is a function from $\square \mathbb{R}^{n}$ to $\{0,1\}$, where 0 and 1 stand for false and true, respectively. An example of interval constraint is

$C([\mathbf{x}]):\left[x_{1}\right] \subset\left[x_{2}\right]$,

where $[\mathbf{x}]=\left[x_{1}\right] \times\left[x_{2}\right]$. An interval constraint is monotonic if

$[\mathbf{x}] \subset[\mathbf{y}] \Rightarrow(C([\mathbf{x}]) \Rightarrow C([\mathbf{y}]))$.

For instance $C([x]) \stackrel{\text { def }}{=} t(0 \in[x])$ is monotonic. 


\subsection{Intervalization function}

Define the intervalization function $l$ as follows:

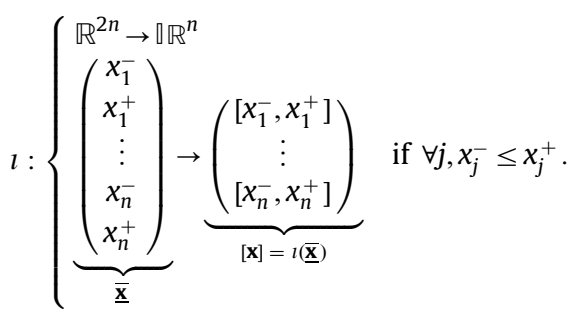

The domain of $l$ is

$\operatorname{dom}(l)=\left\{\underline{\underline{\mathbf{X}}}=\left(x_{1}^{-}, x_{1}^{+}, \ldots, x_{n}^{-}, x_{n}^{+}\right), \forall j, x_{j}^{-} \leq x_{j}^{+}\right\}=l^{-1}\left(\mathbb{Q} \mathbb{R}^{n}\right)$.

Note that the bijection $\imath$ between $\operatorname{dom}(l)$ and $\square \mathbb{R}^{n}$ is a classical and useful conceptual tool (see Kulpa, 2006) to develop original interval algorithms. To an interval constraint $C([\mathbf{x}])$ from $\square \mathbb{R}^{n}$ to $\{0,1\}$ we can define the corresponding bound constraint $\underline{\bar{C}}(\underline{\overline{\mathbf{x}}})=\operatorname{C} \circ l(\underline{\overline{\mathbf{x}}})$ on the bound vector $\underline{\overline{\mathbf{x}}} \in \mathbb{R}^{2 n}$ :

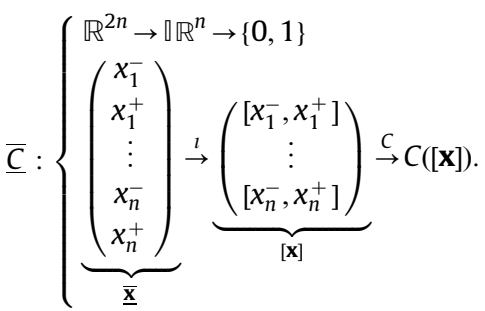

For instance, to the interval constraint

$C([\mathbf{x}]) \stackrel{\text { def }}{=}\left(\left[x_{1}\right] \subset\left[x_{2}\right]\right)$,

we associate the following bound constraint:

$\underline{\bar{C}}\left(\begin{array}{l}x_{1}^{-} \\ x_{1}^{+} \\ x_{2}^{-} \\ x_{2}^{+}\end{array}\right):\left\{\begin{array}{l}x_{1}^{-} \geq x_{2}^{-} \quad \text { and } \\ x_{1}^{+} \leq x_{2}^{+} \quad \text { and } \\ x_{1}^{-} \leq x_{1}^{+} \quad \text { and } \\ x_{2}^{-} \leq x_{2}^{+} .\end{array}\right.$

Symbolic intervals, to be presented in the following section, is a new symbolic tool that makes possible to transform interval problems into a problem on the bounds of the intervals.

\section{Symbolic intervals}

\subsection{Definition}

A symbolic interval is a pair, denoted by $\left[\mathcal{A}^{-}, \mathcal{A}^{+}\right]$, of two mathematical expression $\mathcal{A}^{-}$and $\mathcal{A}^{+}$. For instance

$\left[\sin (a+b), a^{2}+b\right]$

is a symbolic interval. Its lower bound is the expression $\mathcal{A}^{-}=\sin (a+b)$ and its upper bound is the expression $\mathcal{A}^{+}=a^{2}+b$.

\subsection{Operations}

We define the operations on symbolic intervals as classical interval operations, but in a symbolic way.

$$
\begin{aligned}
& {\left[\mathcal{A}^{-}, \mathcal{A}^{+}\right]+\left[\mathcal{B}^{-}, \mathcal{B}^{+}\right]=\left[\mathcal{A}^{-}+\mathcal{B}^{-}, \mathcal{A}^{+}+\mathcal{B}^{+}\right], } \\
& {\left[\mathcal{A}^{-}, \mathcal{A}^{+}\right]-\left[\mathcal{B}^{-}, \mathcal{B}^{+}\right]=\left[\mathcal{A}^{-}-\mathcal{B}^{+}, \mathcal{A}^{+}-\mathcal{B}^{-}\right], } \\
& {\left[\mathcal{A}^{-}, \mathcal{A}^{+}\right] *\left[\mathcal{B}^{-}, \mathcal{B}^{+}\right]=} {\left[\min \left(\mathcal{A}^{-} * \mathcal{B}^{-}, \mathcal{A}^{-} * \mathcal{B}^{+}, \mathcal{A}^{+} * \mathcal{B}^{-}, \mathcal{A}^{+} * \mathcal{B}^{+}\right),\right.} \\
&\left.\max \left(\mathcal{A}^{-} * \mathcal{B}^{-}, \ldots\right)\right],
\end{aligned}
$$

$\left[\mathcal{A}^{-}, \mathcal{A}^{+}\right]^{2}=\left[\max \left(0, \operatorname{sign}\left(\mathcal{A}^{-} * \mathcal{A}^{+}\right)\right) \min \left(\left(\mathcal{A}^{-}\right)^{2},\left(\mathcal{A}^{+}\right)^{2}\right), \max \left(\left(\mathcal{A}^{-}\right)^{2},\left(\mathcal{A}^{+}\right)^{2}\right)\right]$, $\exp \left(\left[\mathcal{A}^{-}, \mathcal{A}^{+}\right]\right)=\left[\exp \left(\mathcal{A}^{-}\right), \exp \left(\mathcal{A}^{+}\right)\right]$

$1 /\left[\mathcal{A}^{-}, \mathcal{A}^{+}\right]=\left[\min \left(1 / \mathcal{A}^{+}, \infty *\left(\mathcal{A}^{-} * \mathcal{A}^{+}\right)\right), \max \left(1 / \mathcal{A}^{-},-\infty *\left(\mathcal{A}^{-} * \mathcal{A}^{+}\right)\right)\right]$,

$\left[\mathcal{A}^{-}, \mathcal{A}^{+}\right] \cap\left[\mathcal{B}^{-}, \mathcal{B}^{+}\right]=\left[\max \left(\mathcal{A}^{-}, \mathcal{B}^{-}\right), \min \left(\left(\mathcal{A}^{+}, \mathcal{B}^{+}\right)\right)\right]$,

$\left[\mathcal{A}^{-}, \mathcal{A}^{+}\right] \sqcup\left[\mathcal{B}^{-}, \mathcal{B}^{+}\right]=\left[\min \left(\mathcal{A}^{-}, \mathcal{B}^{-}\right), \max \left(\left(\mathcal{A}^{+}, \mathcal{B}^{+}\right)\right)\right]$,

$w\left(\left[\mathcal{A}^{-}, \mathcal{A}^{+}\right]\right)=\mathcal{A}^{+}-\mathcal{A}^{-}$.

For instance,

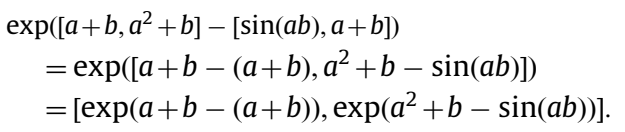

Note that some operations on symbolic intervals have not the same form as their classical numerical counterpart. They have been rewritten in order to get a symbolic interval as the result of the operation, i.e., a pair of two symbolic expressions. For instance, the definition of the square of an interval $[a, b]$ is classically defined by

$[a, b]^{2}=\left[0, \max \left(a^{2}, b^{2}\right)\right] \quad$ if $0 \in[a, b]$

$$
=\left[\min \left(a^{2}, b^{2}\right), \max \left(a^{2}, b^{2}\right)\right] \quad \text { if } 0 \notin[a, b] .
$$

Now, since

$$
\begin{aligned}
\max (0, \operatorname{sign}(a b))=0 & \text { if } 0 \in[a, b] \\
=1 & \text { if } 0 \notin[a, b],
\end{aligned}
$$

we can define $[a, b]^{2}$ by a single expression

$[a, b]^{2}=\left[\max (0, \operatorname{sign}(a b)) \min \left(a^{2}, b^{2}\right), \max \left(a^{2}, b^{2}\right)\right]$.

This explains the definition of $\left[\mathcal{A}^{-}, \mathcal{A}^{+}\right]^{2}$ for symbolic intervals.

\subsection{Relations}

We also extend classical interval relations to symbolic intervals:

$\left(\left[\mathcal{A}^{-}, \mathcal{A}^{+}\right]=\left[\mathcal{B}^{-}, \mathcal{B}^{+}\right]\right) \Leftrightarrow \mathcal{A}^{-}-\mathcal{B}^{-}=0, \quad \mathcal{A}^{+}-\mathcal{B}^{+}=0$

$\left(\left[\mathcal{A}^{-}, \mathcal{A}^{+}\right] \subset\left[\mathcal{B}^{-}, \mathcal{B}^{+}\right]\right) \Leftrightarrow \mathcal{A}^{-}-\mathcal{B}^{-} \geq 0, \quad \mathcal{B}^{+}-\mathcal{A}^{+} \geq 0$.

For instance

$\left(\left[a+b, a^{2}+b\right]=[\sin (a b), a-b]\right)$

$\Leftrightarrow\left(a+b=\sin (a b)\right.$ and $\left.a^{2}+b=a-b\right)$.

Another example is the following:

$\left([a, b] \subset[a, b]^{2}\right) \Leftrightarrow\left\{\begin{array}{l}a-\max (0, \operatorname{sign}(a \cdot b)) * \min \left(a^{2}, b^{2}\right) \geq 0, \\ \max \left(a^{2}, b^{2}\right)-b \geq 0 .\end{array}\right.$

\subsection{Simplification}

The expression involved as lower and upper bound of symbolic intervals are classical expressions over real variables. Thus classical simplification techniques can be applied to symbolic interval. For instance, we can write

$\left[a-b+a+b, a^{2}+c-a * a\right]=[2 a, c]$.

But note that

$[a, b]-[a, b]=[a-b, b-a]$

which is not $[0,0]$. The simplifications can only be performed on the expressions inside the interval and not on the symbolic interval operations. 


\section{Applications}

This section provides some simple potential applications of symbolic intervals.

\subsection{Experimental design}

Assume we want to design an experiment to measure indirectly a quantity $y$ which satisfies the relation

$y=f\left(x_{1}, \ldots, x_{n}\right)$

where the $x_{i}$ can be measured with a accuracy lower than $\varepsilon_{x}$. We also assume that an inclusion function

$[f]:\left\{\begin{array}{l}\square \mathbb{R}^{n} \rightarrow \mathbb{R} \mathbb{R} \\ {[\mathbf{x}] \rightarrow[f]([\mathbf{x}])}\end{array}\right.$

for $f$ is available. Once the interval measurements $\left[x_{i}\right]$ will be collected, this inclusion function will be used to get an interval $[y]$ for $y$. We would like to know with which accuracy $y$ will be obtained, i.e., what will be the size of $[y]=[f]\left(\left[x_{1}\right], \ldots,\left[x_{n}\right]\right)$ in the worst-case? This problem can be formulated as follows:

$\max w([f]([\mathbf{x}]))$.

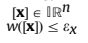

As an illustration, consider the situation where $f(x)=\exp \left(x-x^{2}\right)$, $[f]([x])=\exp \left([x]-[x]^{2}\right)$, and $\varepsilon_{x}=1$. We get the following interval optimization problem:

$\max _{[x] \in \mathbb{R}} w\left(\exp \left([x]-[x]^{2}\right)\right)$.

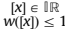

Using the symbolic interval arithmetic, with $[x]=[a, b]$, we get

$\max _{b-a \in[0,1]} e^{b-\max \left(0, \operatorname{sign}(a b) \cdot \min \left(a^{2}, b^{2}\right)\right)}-e^{a-\left(\max \left(a^{2}, b^{2}\right)\right.}$.

Using an interval method, we get that the maximum is inside [3.324807; 3.324808] and the global optimizer satisfies

$\left(a^{*}, b^{*}\right) \in[0.547,0.548] \times[1.547,1.548]$,

i.e., the interval optimizer is an interval $\left[a^{*}, b^{*}\right]$ which satisfies the previous relation. In an estimation point of view, it means that the worst case that could happen is to have an interval measurement $[x] \simeq[0.547,1.547]$. However, in any situation, the accuracy for $y$ will always be better than 3.325 .

Fig. 1 right, shows the set of intervals

$\mathbb{S}=\left\{(a, b) \in[-2,2] \times[-2,2], w([a, b]) \leq 1\right.$ and $\left.w\left(\exp \left([a, b]-[a, b]^{2}\right)\right)>1\right\}$.

The light gray area has been proven to be inside $\mathbb{S}$ and the dark gray area is outside $\mathbb{S}$. The dashed zone corresponds to all pairs $(a, b)$ such that $a>b$, i.e., $[a, b]$ cannot be an interval. The left part of the figure shows the contractions/bisections made by the numerical interval algorithm.

\subsection{Comparing two inclusion functions}

When implementing an interval method to solve a nonlinear problem (optimization, set inversion, etc.), we have to implement an inclusion function for a function $f$ involved in the problem. In practice, $f$ can be described by different expressions each of them producing a different inclusion functions. Denote by $[f]$ and $[g]$ two inclusion functions for $f$. Two situations could appear. (i) One is always more accurate than the other (i.e., $[f] \subset[g]$ or $[g] \subset[f]$ ) and thus, the choice for the inclusion function to be implemented is easy. (ii) For some boxes $[\mathbf{a}],[f]([\mathbf{a}]) \subset[g]([\mathbf{a}])$ and for some other boxes $[\mathbf{b}],[g]([\mathbf{b}]) \subset[f]([\mathbf{b}])$ and it can be worthwhile the implement the intersection $[h]=[g] \cap[f]$. To make such a choice, a comparison of the two inclusion function $[f]$ and $[g]$ is needed and, to our knowledge, no tool exists in the literature to perform such a comparison.

Let us now show how such an intersection can be done using symbolic intervals on a simple example where two inclusion functions $[f]$ and $[g]$ for $f(x)=x^{2}-x$ are available:

$[f]([x])=[x] *([x]-1)$,

$[g]([x])=[x]^{2}-[x]$.

We would like to know for which interval $[x]=[a, b]$, $[f]$ is more accurate than $[g]$. We have

$$
\begin{aligned}
{[f]([x])=} & {[a, b] *([a, b]-1) } \\
= & {[a, b] *[a-1, b-1] } \\
= & {[\min (a(a-1), b(a-1), a(b-1), b(b-1)),} \\
& \max (a(a-1), b(a-1), a(b-1), b(b-1))]
\end{aligned}
$$

and

$$
\begin{aligned}
{[g]([x]) } & =[a, b]^{2}-[a, b] \\
& =\left[\max \left(0, \operatorname{sign}(a \cdot b) \min \left(a^{2}, b^{2}\right)\right), \max \left(a^{2}, b^{2}\right)\right]-[a, b] \\
& =\left[\max \left(0, \operatorname{sign}(a \cdot b) \min \left(a^{2}, b^{2}\right)-b, \max \left(a^{2}, b^{2}\right)-a\right] .\right.
\end{aligned}
$$

Thus

\section{$[f]([x]) \subset[g]([x])$}

$$
\begin{aligned}
& \Leftrightarrow\left\{\begin{array}{l}
\min (a(a-1), b(a-1), a(b-1), b(b-1)) \geq \max \left(0, \operatorname{sign}(a \cdot b) \min \left(a^{2}, b^{2}\right)\right)-b, \\
\max (a(a-1), b(a-1), a(b-1), b(b-1)) \leq \max \left(a^{2}, b^{2}\right)-a
\end{array}\right. \\
& \Leftrightarrow\left\{\begin{array}{l}
\min (a(a-1), b(a-1), a(b-1), b(b-1))-\max \left(0, \operatorname{sign}(a \cdot b) \min \left(a^{2}, b^{2}\right)\right)+b \geq 0, \\
\max \left(a^{2}, b^{2}\right)-a-\max (a(a-1), b(a-1), a(b-1), b(b-1)) \geq 0 .
\end{array}\right.
\end{aligned}
$$

The solution set is represented in Fig. 2, right. On the light gray area are represented the intervals $[x]$ such that $[f]([x]) \subset[g]([x])$ and in the dark gray area are the intervals $[x]$ such that $[f]([x]) \not \subset[g]([x])$. Nothing is known about the intervals in the white area. The
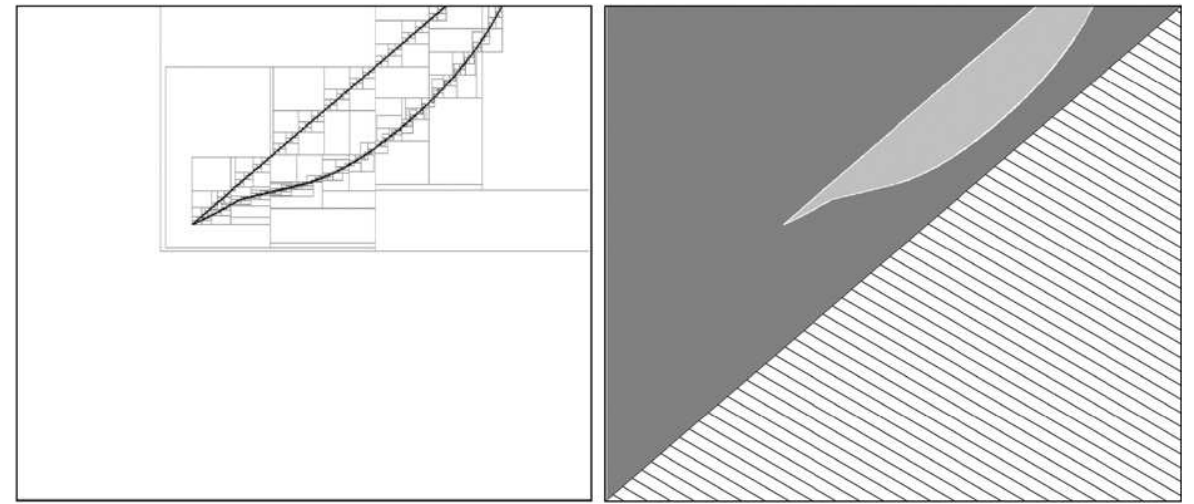

Fig. 1. Left: contractions produced by the numerical interval algorithm. Right: set of all interval $[a, b]$ that belong to $\mathbb{S}$. 

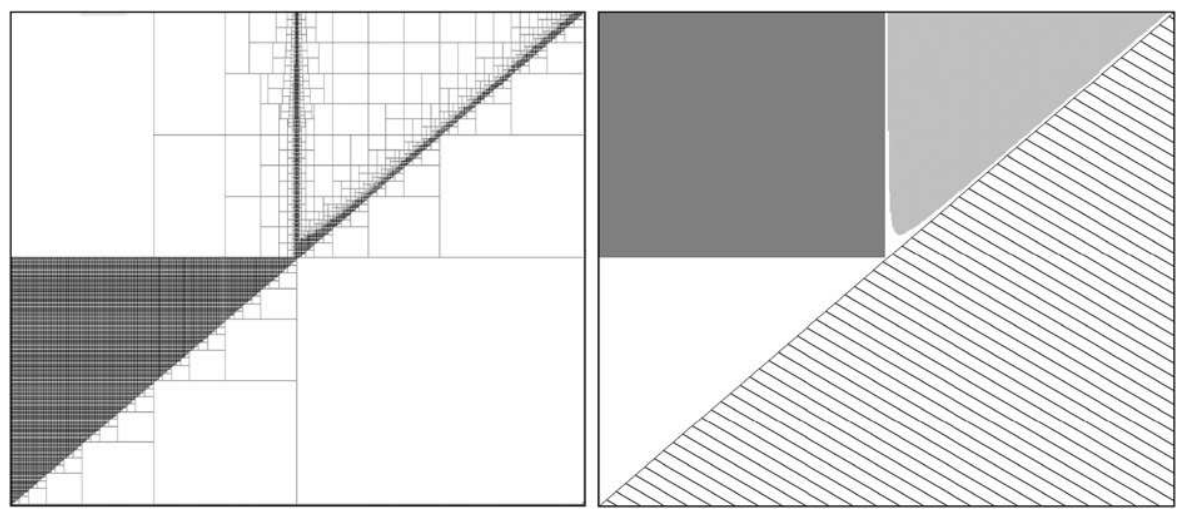

Fig. 2. Comparison between two inclusion functions.
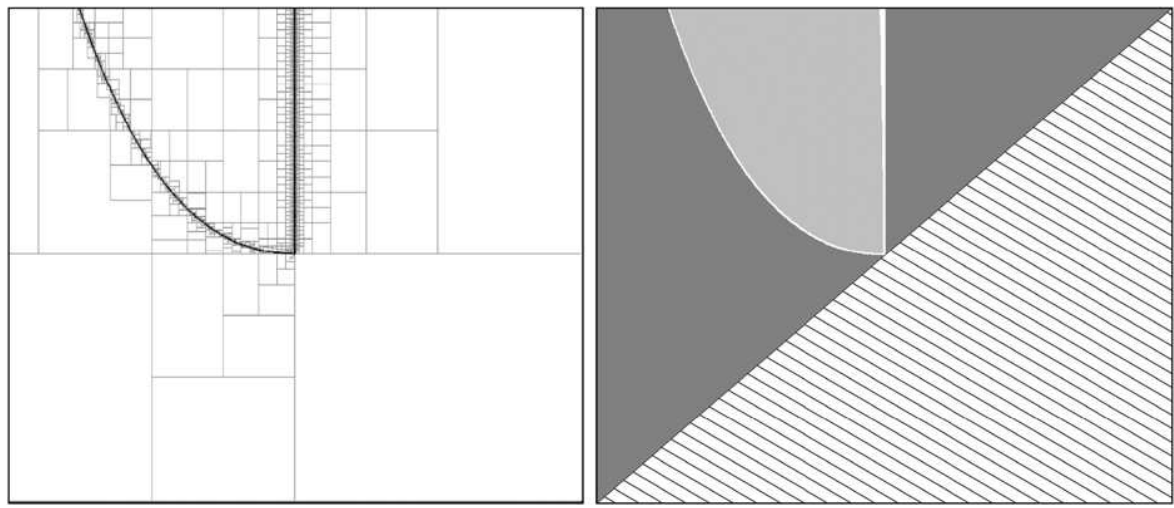

Fig. 3. Left: contractions made by the numerical interval algorithm. Right: set of intervals that are contracted by the Newton contractor. The frame box is $[-2,2] \times[-2,2]$.

hatshed zone are points $(a, b)$ which do not correspond to intervals $[a, b]$.

The table below provides a comparison for three intervals.

\begin{tabular}{llll}
\hline$[x]$ & {$[f]([x])$} & {$[g]([x])$} & Relation \\
\hline$[1,2]$ & {$[0,2]$} & {$[-1,3]$} & {$[f]([x]) \subset[g]([x])$} \\
{$[-2,-1]$} & {$[1,6]$} & {$[2,6]$} & {$[f]([x]) \subset[g]([x])$} \\
{$[-1,1]$} & {$[-2,2]$} & {$[-1,2]$} & {$[g]([x]) \subset[f]([x])$} \\
\hline
\end{tabular}

Note that for $[x]=[-2,-1]$, we have $[f]([x]) \subset[g]([x])$, but we are in the undetermined area (white). The inclusion was not proven by the method because we do not have a strict inclusion (i.e., $\forall \varepsilon>0,[1,6]+[-\varepsilon, \varepsilon]$ is not a subset of $[2,6])$.

\subsection{Analysis of the Newton contractor}

The previous section has shown how two inclusion functions could be compared. The same principle could be used to compare two contractors. Here, we shall see how symbolic intervals can be used to analyze the behavior of contractors. More precisely, we would like to characterize the set of all intervals $[x]$ that can be contracted by a given contractor. As an illustration, we consider the equation $f(x)=0$ with $f(x)=e^{x}-1$. The associated interval Newton contractor is defined by

$\mathcal{N}([x])=x_{0}-\frac{f\left(x_{0}\right)}{\left[f^{\prime}\right]([x])}$, where $x_{0}$ is any point in $[x]$. Here, we shall take $x_{0}=x^{-}$and thus

$\mathcal{N}([x])=x^{-}-\frac{f\left(x^{-}\right)}{\left[f^{\prime}\right]([x])}=x^{-}-\frac{e^{x^{-}}-1}{\exp \left(\left[x^{-}, x^{+}\right]\right)}$.

The Newton operator is contracting if

$\mathcal{N}([x]) \subset[x]$.

Let us compute the set of all intervals such that $\mathcal{N}$ is contracting. If we set $[x]=[a, b]$, we get

$\mathcal{N}([a, b])=a-\frac{a-1}{\exp ([a, b])}$.

Using symbolic interval arithmetic, the relation $\mathcal{N}([a, b]) \subset[a, b]$ transforms into

$\left\{\begin{array}{l}a-\max \left(\frac{e^{a}-1}{e^{b}}, \frac{e^{a}-1}{e^{a}}\right)-a \geq 0, \\ b-a+\min \left(\frac{e^{a}-1}{e^{b}}, \frac{e^{a}-1}{e^{a}}\right) \geq 0, \\ b-a \geq 0 .\end{array}\right.$

Fig. 3 represents the set $\mathbb{S}$ all pairs $(a, b)$ which satisfy this set of constraints. The light gray area is proven to be inside $\mathbb{S}$ and the dark gray area corresponds to pairs $(a, b)$ outside $\mathbb{S}$.

\subsection{Proving the asymptotic optimality of a contractor}

Consider a contractor $\mathcal{C}$ and denote by $\mathbb{S}$ the set of all points $\mathbf{x}$ such that $\mathcal{C}(\{\mathbf{x}\})=\{\mathbf{x}\}$. The contractor is asymptotically optimal if $\forall[\mathbf{x}] \in \mathbb{\square} \mathbb{R}^{n}, \quad \mathcal{C}([\mathbf{x}])=[\mathbb{S} \cap[\mathbf{x}]]$, 
where $[S \cap[\mathbf{x}]]$ denotes the smallest box which encloses $\mathbb{S} \cap[\mathbf{x}]$. When dealing with constraint satisfaction problem (CSP) (Sam-Haroud and Faltings, 1996), it can be useful to prove that a given contractor $\mathcal{C}$ is asymptotically optimal, but to our knowledge, no methodology exists in the literature to prove it. In this section, we show that symbolic intervals could be helpful for that. To illustrate the principle, consider an equation

$x^{2}-x=0$.

A hull consistency contractor amounts to iterate the following statements:

$[x]:=[x] \cap[x]^{2}$,

$[x]:=[x] \cap \sqrt{[x]}$,

from an initial interval $[x](0)$ until a steady interval is reached. The methodology we propose to prove that the resulting contractor is asymptotically optimal it is described below.

Step 1. Compute all solutions of the equation $x^{2}-x=0$. With an interval method (with bisections), we get that we have exactly two solutions $x_{1}=0$ and $x_{2}=1$. Thus we know any contractor associated with the constraint $x^{2}-x=0$ has at least three steady boxes (those corresponding to $[0,0],[1,1],[0,1]$ ).

Step 2 . Since the contractor will converge the biggest box inside $[x](0)$ which satisfies

$\left\{\begin{array}{l}{[x] \subset[x]^{2}} \\ {[x] \subset \sqrt{[x]}}\end{array}\right.$

The interval CSP translates into the equivalent bound CSP

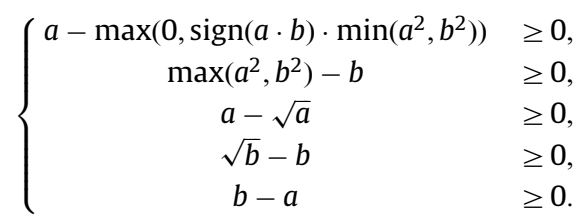

Let us compute all solutions of this bound system. We get the three following boxes for $(a, b)$ :

$[0.999999999999,1] \times[0.999999999999,1]$,

$\left[0,3 \times 10^{-39}\right] \times\left[0,3 \times 10^{-39}\right]$

$\left[0,3 \times 10^{-39}\right] \times[0.999999999999,1]$.

A unicicity test can concludes about that each of the three boxes contain a unique solution. Thus, we know that we have exactly three steady boxes. Thus, we have proven that the hull contractor is asymptotically optimal.

\subsection{Quantifier elimination}

In this section, we will show that symbolic interval arithmetic can be used for quantifier elimination (see e.g., Collins, 1975). Consider the quantified formula

$\exists \mathbf{y} \in[\mathbf{y}] \subset \mathbb{R}^{m}, \quad f(\mathbf{p}, \mathbf{y})=0$,

where $\mathbf{p} \in \mathbb{R}^{n}$ and $f: \mathbb{R}^{n} \times \mathbb{R}^{m} \rightarrow \mathbb{R}$ is assumed to be continuous. Assume that in the expression of $f$, the $y_{i}$ 's occur only once. Denote by $[f](\mathbf{p},[\mathbf{y}])$ the natural inclusion function for $f$ with respect to $\mathbf{y}$. From Moore (1979), we have

$[f](\mathbf{p},[\mathbf{y}])=\{f(\mathbf{p}, \mathbf{y}), \mathbf{y} \in[\mathbf{y}]\}$,

and thus formula (15) is equivalent to

$0 \in[f](\mathbf{p},[\mathbf{y}])$.

Using symbolic interval arithmetic, we are able to compute an expression $\alpha(\mathbf{p})$ and $\beta(\mathbf{p})$ for the bounds of $[f](\mathbf{p},[\mathbf{y}])$. As a consequence, Formula (15) is equivalent to

$\alpha(\mathbf{p}) \leq 0 \leq \beta(\mathbf{p})$,

where no quantifier appears anymore. Note that similar reasoning can be found in Gardenes et al. (1985) and Goldsztejn (2005) in the context of modal interval analysis. As an illustration, consider the quantified distance problem (Grandon and Goldsztejn, 2006). The set to be characterized is defined by

$\mathbb{S}=\{\mathbf{p} \in \mathbb{R}^{2}, \exists \mathbf{y} \in[\mathbf{y}], \underbrace{\left(p_{1}-y_{1}\right)^{2}+\left(p_{2}-y_{2}\right)^{2}-y_{3}}_{f(\mathbf{p}, \mathbf{y})}=0\}$,

where

$[\mathbf{y}]=[-0.5,0.5] \times[-0.5,1.3] \times[1.69,2.56]$

Here $\mathbb{S}$ represents the set of all feasible $\mathbf{p}$ that are at a distance $\sqrt{y_{3}}$ from a point $\left(y_{1}, y_{2}\right)$, when the $\mathbf{y}=\left(y_{1}, y_{2}, y_{3}\right) \in[\mathbf{y}]$. We have

$$
\begin{aligned}
{[f](\mathbf{p},[\mathbf{y}])=} & \left(p_{1}-\left[y_{1}\right]\right)^{2}+\left(p_{2}-\left[y_{2}\right]\right)^{2}-\left[y_{3}\right] \\
= & {\left[\operatorname { m a x } ( 0 , \operatorname { s i g n } ( ( p _ { 1 } - y _ { 1 } ^ { + } ) ( p _ { 1 } - y _ { 1 } ^ { - } ) ) ) \operatorname { m i n } \left(\left(p_{1}-y_{1}^{+}\right)^{2},\right.\right.} \\
& \left.\left(p_{1}-y_{1}^{-}\right)^{2}\right)+\max \left(0, \operatorname{sign}\left(\left(p_{2}-y_{2}^{+}\right)\left(p_{2}-y_{2}^{-}\right)\right)\right) \min \left(\left(p_{2}-y_{2}^{+}\right)^{2},\right. \\
& \left.\left(p_{2}-y_{2}^{-}\right)^{2}\right)-y_{3}^{+}, \max \left(\left(p_{1}-y_{1}^{+}\right)^{2},\right. \\
& \left.\left.\left(p_{1}-y_{1}^{-}\right)^{2}\right)+\max \left(\left(p_{2}-y_{2}^{+}\right)^{2},\left(p_{2}-y_{2}^{-}\right)^{2}\right)-y_{3}^{-}\right] .
\end{aligned}
$$

Therefore, the set $\mathbb{S}$ can be defined by the quantifier free formula

$$
\left\{\begin{array}{l}
\max \left(0, \operatorname{sign}\left(\left(p_{1}-0.5\right)\left(p_{1}+0.5\right)\right)\right) \min \left(\left(p_{1}-0.5\right)^{2},\left(p_{1}+0.5\right)^{2}\right) \\
\quad+\max \left(0, \operatorname{sign}\left(\left(p_{2}-1.3\right)\left(p_{2}+0.5\right)\right)\right) \min \left(\left(p_{2}-1.3\right)^{2},\left(p_{2}+0.5\right)^{2}\right) \leq 2.56, \\
\max \left(\left(p_{1}-0.5\right)^{2},\left(p_{1}+0.5\right)^{2}\right)+\max \left(\left(p_{2}-1.3\right)^{2},\left(p_{2}+0.5\right)^{2}\right) \geq 1.69
\end{array}\right.
$$

It can thus be characterized by an interval set inversion algorithm (Jaulin et al., 2001). Fig. 4 have been computed in $0.4 \mathrm{~s}$ in a
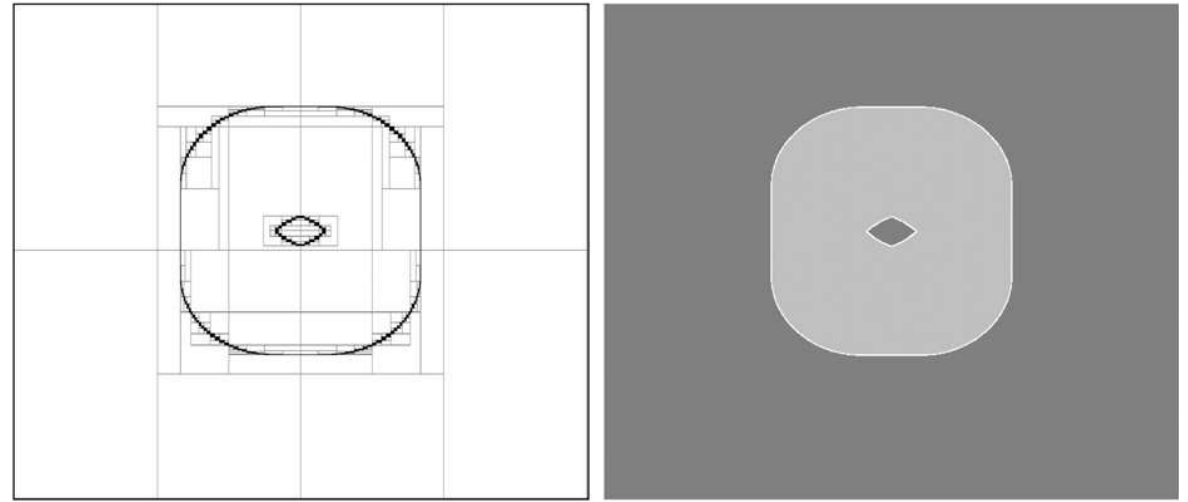

Fig. 4. Left: contractions performed by the interval agorithm. Right: solution set of the quantified distance problem. 
standard laptop. The left subfigure shows all contractions that have been performed by the algorithm and the right subfigure represents the set $\mathbb{S}$. The light gray area is proven to be inside $\mathbb{S}$ and the dark gray area is outside $\mathbb{S}$. The results are very similar to those obtained in Grandon and Goldsztejn (2006) where generalized interval computation has been used to eliminate the quantifier. Note that the symbolic interval approach made possible to have inner contractions which is not possible with the approach proposed in Grandon and Goldsztejn (2006).

In this section, we have shown for the first time that interval arithmetic as defined by Moore (1979) and applied in a symbolic way, can be an efficient tool to deal with some specific symbolic quantifier elimination problem.

Remark. The formula

$\forall \mathbf{y} \in[\mathbf{y}], \quad \exists \mathbf{x} \in[\mathbf{x}], \quad f(\mathbf{p}, \mathbf{x}, \mathbf{y})=0$,

can also be transformed into one inequality of the form $g(\mathbf{p}) \leq 0$, if $f$ is continuous and if, in the expression of $f$, all $x_{i}$ and all $y_{j}$ occur only once. This can be done by using modal interval arithmetic in a symbolic way. To each variable related to a subexpression of $a$, we associate (Vinas et al., 2006) the pair (also called modal interval)

$\left[\max _{\mathbf{y} \in[\mathbf{y}]]} \min _{\mathbf{x} \in[\mathbf{x}]} a(\mathbf{p}, \mathbf{x}, \mathbf{y}), \min _{\mathbf{y} \in[\mathbf{y}]} \max _{\mathbf{x} \in[\mathbf{x}]} a(\mathbf{p}, \mathbf{x}, \mathbf{y})\right]$.

An arithmetic can be developed on these modal intervals. By applying symbolically the modal interval arithmetic on $f$, we obtain a symbolic expression for

$$
\alpha(\mathbf{p})=\max _{\mathbf{y} \in[\mathbf{y}]} \min _{\mathbf{x} \in[\mathbf{x}]} f(\mathbf{p}, \mathbf{x}, \mathbf{y}) \quad \text { and } \quad \beta(\mathbf{p})=\min _{\mathbf{y} \in[\mathbf{y}]} \max _{\mathbf{x} \in[\mathbf{x}]} f(\mathbf{p}, \mathbf{x}, \mathbf{y})
$$

and thus Eq. (21) transforms into the following quantifier free formula:

$\alpha(\mathbf{p}) \leq 0 \quad$ and $\quad \beta(\mathbf{p}) \geq 0$.

\section{Conclusion}

This paper deals with interval problems, where the solution set is a set of intervals. The main contribution of the paper is the introduction of symbolic intervals with the corresponding operations. This new tool makes it possible to transform in an easy way an interval problem into a punctual nonlinear problem on the bounds of the intervals. This nonlinear problem on the bounds can then be solved using classical interval analysis methods. The approach that has been proposed to solve nonlinear interval problems applies interval calculus concepts twice: the first time in a symbolic way and the second time in a numerical way. This makes it possible to solve a huge class of nonlinear interval problems that cannot be solved without this approach. To illustrate the efficiency and the interest of symbolic intervals, several applications have been considered.

\section{References}

Collins, G., 1975. Quantifier elimination for real closed fields by cylindrical algebraic decomposition. In: Second GI Conference on Automata Theory and Formal Languages, Lecture Notes in Computer Science, vol. 33, Kaiserslauten, pp. 134-183.

Gardenes, E., Mielgo, H., Trepat, A., 1985. Modal intervals: reasons and ground semantics. In: Nickel, K. (Ed.), Interval Mathematics 1985, vol. 212. Springer, Berlin, Germany, pp. 27-35.

Goldsztejn, A., 2005. Définition et Applications des Extensions des Fonctions Réelles aux Intervalles Généralisés. Ph.D. Thesis, Université de Nice-Sophia Antipolis.

Grandon, C., Goldsztejn, A., 2006. Quantifier elimination versus generalized interval evaluation: a comparison on a specific class of quantified constraint. In: Proceedings of the 11th International Conference on Information Processing and Management of Uncertainty in Knowledge-Based Systems, Paris, France, July 2006.

Hansen, E.R., 1992. Bounding the solution of interval linear equations. SIAM Journal on Numerical Analysis 29 (5), 1493-1503.

Jaulin, L., Kieffer, M., Didrit, O., Walter, E, 2001. Applied Interval Analysis, with Examples in Parameter and State Estimation, Robust Control and Robotics. Springer, London.

Kulpa, Z., 2006. Diagrammatic interval analysis with applications. IPPT PAN Reports (ISSN 0208-5658), Warsaw 2006.

Moore, R.E., 1979. Methods and Applications of Interval Analysis. SIAM, Philadelphia, PA.

Sam-Haroud, D., Faltings, B., 1996. Consistency techniques for continuous constraints. Constraints 1 (1-2), 85-118.

van Emden, M., 1999. Algorithmic power from declarative use of redundant constraints. Constraints 4 (4), 363-381.

Vinas, P.H., Sainz, M.A., Vehi, J., Jaulin, L., 2006. Quantified set inversion algorithm with applications to control. Reliable Computing 11 (5), 369-382. 